\title{
Inhibition of proliferation of estrogen receptor-positive MCF-7 human breast cancer cells by tamoxifen through c-Jun transcription factors
}

\author{
YAN XU ${ }^{1 *}$, SHI-TAO ZOU ${ }^{2 *}$, RAN ZHU $^{3}$, WEI LI ${ }^{1}$, CHUN-WEI GU ${ }^{1}$, \\ SHAO-HUA WEI ${ }^{1}$, JIA-MING XIE ${ }^{1}$ and HAO-RONG WU ${ }^{1}$ \\ ${ }^{1}$ Department of General Surgery, Second Affiliated Hospital of Soochow University, Suzhou, Jiangsu 215004; \\ ${ }^{2}$ Institute of Cancer Research, Fourth Affiliated Hospital of Soochow University, Wuxi, Jiangsu 214062; \\ ${ }^{3}$ School of Radiation Medicine and Protection, Medical College, Soochow University, Suzhou, Jiangsu 215123, P.R. China
}

Received October 16, 2012; Accepted January 21, 2013

DOI: $10.3892 / \mathrm{mmr} .2013 .1306$

\begin{abstract}
Activator of protein 1 (AP-1) is a heterodimeric transcription factor composed of various members of the Jun and Fos families and binds to DNA at specific AP-1 binding sites. AP-1 transcriptional activity is increased by phosphorylation at serine residues in the c-Jun component of AP-1. In the present study, the proliferation of MCF-7 breast cancer cells was found to be suppressed by tamoxifen (TAM)-activated c-Jun through the protein kinase C (PKC) pathway. The molecular mechanism by which c-Jun activation induces antiproliferative signals in estrogen receptor (ER)-positive MCF-7 human breast cancer cells remains unknown. TAM inhibited the proliferation of ER-positive MCF-7 human breast cancer cells and ER-negative MDA-MB-435 human breast cancer cells and $48 \mathrm{~h}$ incubation with $10 \mu \mathrm{M}$ TAM led to inhibition of $80 \%$ of proliferation. In addition, no significant difference in c-Jun mRNA and protein levels was detected in MCF-7 and MDA-MB-435 cells stimulated by TAM for $48 \mathrm{~h}$. TAM treatment of MCF-7 cells activated the transcriptional activity of AP-1, which responds specifically to phorbol ester. To determine the role of c-Jun in the antiproliferation of MCF-7 cells stimulated by TAM, the inhibition rates of MCF-7 cells were correlated with c-Jun expression and stimulation of TAM. Results showed that the inhibition rate of TAM-stimulated MCF-7 cells was positively regulated by overexpression of c-Jun and negatively regulated
\end{abstract}

Correspondence to: Professor Hao-Rong Wu, Department of General Surgery, Second Affiliated Hospital of Soochow University, 181 Sanxiang Road, Suzhou, Jiangsu 215004, P.R. China

E-mail: wuhaorong@vip.sina.com

*Contributed equally

Key words: tamoxifen, protein kinase C, c-Jun, antiproliferation, MCF-7 by underexpression of c-Jun. Overall, these results indicate that the TAM-stimulated antiproliferation of MCF-7 cells is positively regulated by c-Jun through activation of the PKC pathway.

\section{Introduction}

Tamoxifen (TAM) is the most commonly prescribed drug for the prevention and treatment of breast cancer $(1,2)$. TAM is a non-steroidal anti-estrogen which acts, at least in part, by compete blockage of the estrogen receptor $(\mathrm{ER})(3,4)$. However, the existence of an alternative mechanism of action for this drug is supported by the following clinical observations: i) $30 \%$ of patients with ER-negative cancer cells respond to TAM and ii) $30 \%$ of patients with ER-positive cancer cells are not sensitive to this anti-estrogen (5). The ER activates transcription from classical hormone response elements, to which the ER binds directly and from various alternative response elements, to which the ER does not bind. ER action upon the ovalbumin proximal promoter and the collagenase and IGF-1 genes traces to activator of protein 1 (AP-1) sites that bind members of the Jun/Fos family of transcription factors $(6,7)$. ER action upon the quinine reductase gene traces to an electrophile response element and these have been reported to bind ATF transcription factors, which are potential dimerization partners with Jun (8). ER action upon the cyclin D gene is linked to a CRE-like element that also binds Jun/ATF (9-11). ER also enhances the activity of promoters that are regulated by other factors.

Protein kinase C (PKC) was originally identified as a phospholipid- and calcium-dependent protein kinase (12). PKC affects diverse cell functions through phosphorylation of target proteins. These cell functions involve a wide variety of fundamental physiological processes, including signal transduction, modulation of gene expression, proliferation and apoptosis (13-15). Individual PKC isozymes exhibit various tissue distributions, subcellular localizations and biochemical properties, an indication that each member of the family plays specialized roles (14), which ultimately translates into unique correlations with disease. 
The pAP-1(PMA)-TA-luc plasmid contains the AP-1 elements, which are designed to monitor the induction of the PKC signal transduction pathway. PKC is involved in various biochemical processes relevant to signal transduction pathways and linked to lipid signaling pathways (15). By contrast, isoenzymes of PKC affect a number of biochemical processes, including cell growth, differentiation and transformation and plays a key role in signal transduction from multiple extracellular receptors $(16,17)$. PKC activity is regulated by multiple activators and cofactors which associate via conserved domains within the enzyme structure (18).

In the present study, TAM was observed to inhibit the grow th of the human ER-positive breast cancer cell line, MCF-7 and ER-negative breast cancer cell line, MDA-MB-435, in a concentration-dependent manner. A previous study reported that the observed effects of TAM and its active metabolite on the proliferation of MDA-MB-435 cells were due to an ER-independent mechanism (5). To verify the role of c-Jun in TAM suppression of proliferation of the two breast cancer cell lines, the expression of c-Jun mRNA and protein levels were examined by reverse transcription polymerase chain reaction (RT-PCR) and western blot analysis. TAM treatment of MCF-7 cells activated the transcriptional activities of AP-1(PMA), which responds specifically to phorbol ester. Results indicate that TAM functions as an AP-1 activator to mediate cell functions. The inhibition rates of MCF-7 cells were found to correlate with c-Jun expression and stimulation of TAM, whereby TAM-stimulated MCF-7 cells were positively regulated by c-Jun overexpression and negatively regulated by c-Jun underexpression. To the best of our knowledge, this is the first study on the correlation between c-Jun and antiproliferation of TAM-stimulated MCF-7 cells.

\section{Materials and methods}

Cell culture. Human breast cancer cell lines, MCF-7 and MDA-MB-435, were obtained from Shanghai Cell Bank (Shanghai, China). Cells were cultured in Dulbecco's modified Eagle's medium (Gibco-BRL, Carlsbad, CA, USA) containing $10 \%$ fetal bovine serum in a humidified atmosphere with $5 \% \mathrm{CO}_{2}$ at $37^{\circ} \mathrm{C}$.

Cell proliferation assays. MCF-7 and MDA-MB-435 cells were plated at $1 \times 10^{4}$ cells/well in 96-well plates with six replicate wells, for $24 \mathrm{~h}$ in a humidified incubator $\left(5 \% \mathrm{CO}_{2}\right.$ at $\left.37^{\circ} \mathrm{C}\right)$. Cells were treated with various concentrations of TAM (The First Affiliated Hospital of Soochow University, Suzhou, China) for $48 \mathrm{~h}$. Following this, $10 \mu \mathrm{l}$ CCK-8 (Dojindo Laboratories, Kumamoto, Japan) solution was added to each well of the plate to measure cell proliferation. The plate was incubated for $4 \mathrm{~h}$ in the incubator. Optical density (OD) was measured at a wavelength of $450 \mathrm{~nm}$ using a microplate reader. Data were presented as the mean $\pm \mathrm{SD}$, which was derived from triplicate samples of at least three independent experiments. To examine the effect of c-Jun on inhibition rates of TAM-stimulated MCF-7 cells, cells were transfected with pIRES2-EGFP-c-Jun or pGPU6/GFP/Neo-c-Jun-shRNA using Lipofectamine 2000 (Invitrogen, Carlsbad, CA, USA). Cells were stimulated with TAM $48 \mathrm{~h}$ post-transfection.
Semiquantitative RT-PCR. Total RNA from TAM-treated or untreated MCF-7 and MDA-MB-435 cells was extracted by TRIzol (Gibco-BRL) according to the manufacturer's instructions. cDNA was generated from total RNA, using M-MLV RT (MBI; Fermentas, Waltham, MA, USA). Amplification was performed over 28 cycles consisting of $94^{\circ} \mathrm{C}$ for $35 \mathrm{sec}, 53^{\circ} \mathrm{C}$ for $45 \mathrm{sec}$ and $72^{\circ} \mathrm{C}$ for $1 \mathrm{~min}$. PCR products were separated by electrophoresis on a $1.5 \%$ agarose gel and stained with ethidium bromide to visualize the bands. To compare differences among samples, the relative intensity of each band was normalized against the intensity of the GAPDH band amplified from the same sample. The primer sequences for the genes and expected product sizes were as follows: 5'-TGAACGGGAAGCTC ACTGG-3' (sense) and 5'-TCCACCACCCTGTTGCTGTA-3 (antisense) for GAPDH (307 bp); 5'-GCCTCAGACAGTGCC CGAGAT-3' (sense) and 5'-GTTTAAGCTGTGCCACC TGTTCC-3' (antisense) for c-Jun (245 bp).

Western blot analysis. MCF-7 and MDA-MB-435 cells were harvested $48 \mathrm{~h}$ after TAM treatment and lysed with SDS sample buffer (80 mM Tris-HCl, 2\% SDS, $300 \mathrm{mM} \mathrm{NaCl}$ and $1.6 \mathrm{mM}$ EDTA). Cell extracts were separated using 10\% SDS-PAGE gel electrophoresis, transferred onto nitrocellulose membrane and blocked with 5\% skimmed milk. Following blocking, membranes were incubated with antibodies against GAPDH or c-Jun and then incubated with HRP-conjugated anti-mouse or -rabbit IgG antibodies. Protein bands were visualized with ECL solution.

Transient expression reporter gene assay. MCF-7 and MDA-MB-435 cells grown in 24-well plates were transfected with $1 \mu \mathrm{g}$ reporter plasmids, pAP-1(PMA)-Luc (Clontech, Palo Alto, CA, USA) or pAP-1-Luc, to investigate the role of TAM in this signaling pathway. Relative luciferase activity was normalized by co-transfection with $50 \mathrm{ng}$ pRL-SV40. The cells were then stimulated with TAM for $36 \mathrm{~h}$. Cell lysates were prepared using the Dual-Luciferase reporter assay system (Promega, Madison, WI, USA) and luciferase activity was measured with GloMax 20/20. Data are presented as mean \pm SD of 3 independent experiments.

\section{Results}

Effect of TAM on the growth of MCF-7 and MDA-MB-435 cells in vitro. To observe the proliferation inhibitory effects of TAM, MCF-7 and MDA-MB-435 cells were treated with various concentrations of TAM $(0,2.5,5$ and $10 \mu \mathrm{M})$ for $48 \mathrm{~h}$ and the rate of proliferation inhibition was detected by CCK-8 assay. As is evident in Fig. $1 \mathrm{~A}$ and B, TAM was found to significantly inhibit the proliferation of MCF-7 and MDA-MB-435 cells. The effect of inhibition was enhanced with increased concentrations of TAM.

Expression of c-Jun in MCF-7 and MDA-MB-435 cells stimulated by TAM. The effect of TAM on expression of c-Jun of MCF-7 and MDA-MB-435 cells was investigated using RT-PCR and western blot analysis. MCF-7 and MDA-MB-435 cells were treated with various concentrations of TAM $(0,2.5$, 5 and $10 \mu \mathrm{M}$ ) for $48 \mathrm{~h}$ and c-Jun mRNA and protein levels were examined by RT-PCR and western blot analysis. No 


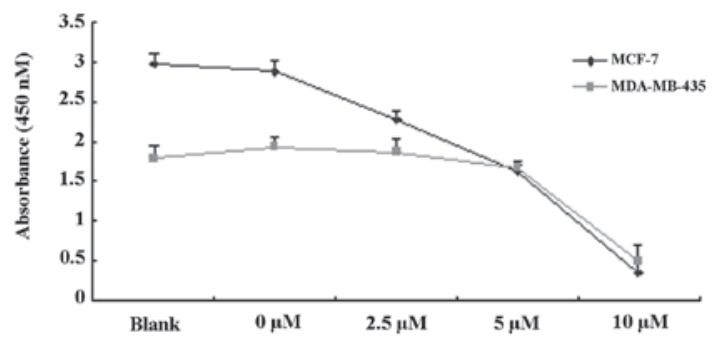

Figure 1. TAM inhibits proliferation of MCF-7 and MDA-MB-435 cells in vitro. MCF-7 and MDA-MB-435 cells were treated with various concentration of TAM for $48 \mathrm{~h}$ and cell growth was assessed by CCK- 8 assay. TAM, tamoxifen.

A

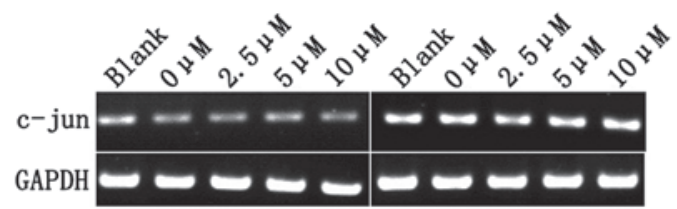

B

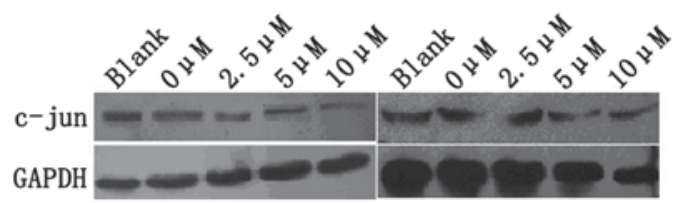

Figure 2. Expression levels of c-Jun in MCF-7 and MDA-MB-435 cells treated with various concentration of TAM. GAPDH was used as an internal contro for loading. c-Jun expression was analyzed by (A) RT-PCR and (B) western blot analysis. No significant changes in MCF-7 and MDA-MB-435 cells were found. TAM, tamoxifen; RT-PCR, reverse transcription-polymerase chain reaction.
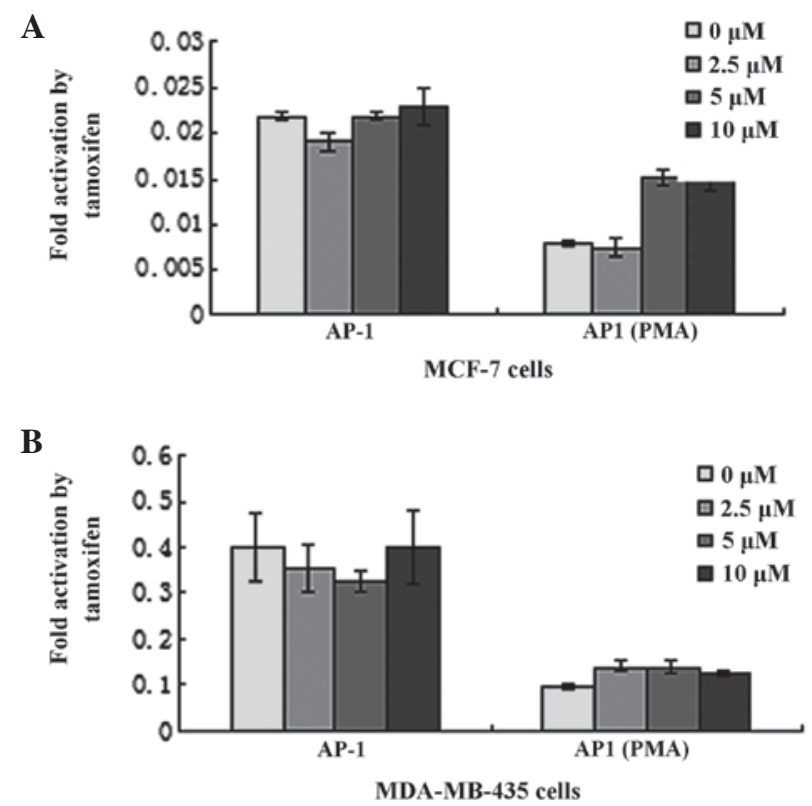

Figure 3. Various concentrations of TAM activate the transcriptional activities of AP-1(PMA) in MCF-7 cells. MCF-7 and MDA-MB-435 cells were transfected with pAP-1(PMA)-Luc or pAP-1-Luc vectors. (A) Dose-dependent activation of TAM on the activity of the reporter gene AP-1(PMA), but not AP-1 in MCF-7 cells. (B) TAM had no significant effect on the activity of the reporter gene AP-1(PMA) and AP-1 in MDA-MB-435 cells. pRL-SV40 encoding Renilla luciferase was used as the internal control. Luciferase activity was determined $36 \mathrm{~h}$ following transfection. Each value represents mean \pm SD of 3 independent experiments. AP-1(PMA), Activator of protein 1 (responds specifically to phorbol ester); TAM, tamoxifen.
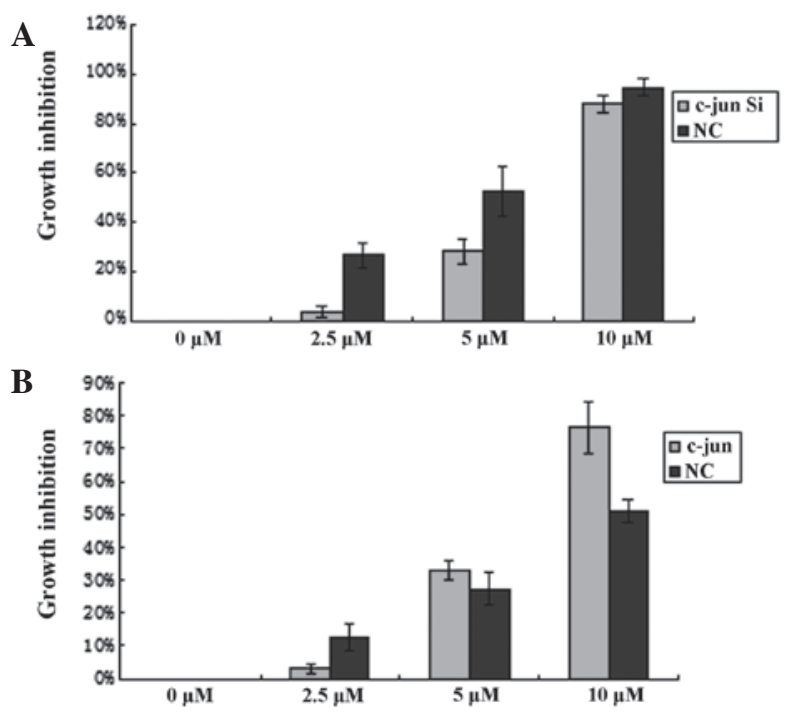

Figure 4. Inhibition rates of TAM-stimulated MCF-7 cells were regulated by c-Jun. (A) Downregulation of c-Jun reduces MCF-7 cell inhibition rates stimulated by TAM in vitro. (B) Upregulation of c-Jun increased MCF-7 cells inhibition rates stimulated by TAM in vitro. TAM, tamoxifen.

significant changes in MCF-7 and MDA-MB-435 cells were observed (Fig. 2A and B).

TAM activates AP-1(PMA)-mediated transcriptional activation in MCF-7 cells. TAM is thought to function primarily by competitive blockage of the ER. In this study, the Dual-Luciferase reporter assay system was utilized to investigate the role of TAM on signaling pathways, including AP-1(PMA) and AP-1. The plasmid pAP-1(PMA)-Luc and pAP-1-Luc were transfected into MCF-7 and MDA-MB-435 cells, which encode luciferase controlled by AP-1(PMA) and AP-1 elements, respectively. Relative luciferase activity was normalized by co-transfection with pRL-SV40. Cells were stimulated with various concentrations of TAM $36 \mathrm{~h}$ post-transfection. In MCF-7 cells, TAM enhanced AP-1(PMA)-luciferase activity by $200 \%$ (Fig. 3A). In addition, TAM was identified to induce dose-dependent activation of the AP-1(PMA) reporter gene. However, TAM had a limited effect on AP-1. In MDA-MB-435 cells, TAM had almost no effect on AP-1(PMA) and AP-1 (Fig. 3B).

Inhibition of proliferation of MCF-7 cells by TAM through c-Jun. To investigate a role for c-Jun in inhibition of TAM-stimulated MCF-7 cell growth, c-Jun vectors with upregulated or downregulated expression of c-Jun in cells were utilized. MCF-7 cells were transfected with pIRES2-EGFP-c-Jun or pGPU6/GFP/Neo-c-Jun-shRNA and stimulated with TAM $48 \mathrm{~h}$ post-transfection. CCK-8 was used to measure cell proliferation. Inhibition rates of TAM-stimulated MCF-7 cells were found to be positively and negatively regulated by overexpression and underexpression of c-Jun, respectively (Fig. 4A and B). These results indicate that c-Jun mediates antiproliferation signals in TAM-stimulated MCF-7 cells and TAM-effected c-Jun may also induce a protein with antiproliferative effects. 


\section{Discussion}

Antiestrogenic drugs, including TAM, provide an effective therapeutic agent for females with hormone-dependent cancer in neoadjuvant, adjuvant and advanced disease settings (19). However, the existence of an alternative mechanism of action for this drug is supported by a number of clinical observations. For example, $30 \%$ of patients with ER-negative cancer cells respond to TAM and $30 \%$ of patients with ER-positive cancer cells are not sensitive to this anti-estrogen (5). Therefore, the uses of TAM are limited. Long-term exposure to TAM is associated with resistance and, in specific cases, with tumor growth stimulation $(20,21)$. The signaling pathways by which breast tumors acquire the ability to grow in the presence of antiestrogens, including TAM, remain poorly understood. One of the mechanisms by which cell lines develop resistance to antiestrogens is through the utilization of alternative signaling pathways that support cell growth in the presence of antiestrogens. Several signalling molecules, including PKC- $\delta$, are known to play a major role in E2-mediated signaling $(22,23)$.

The PKC family of serine/threonine kinases has been intensively studied in cancer since their identification as major receptors for the tumor-promoting phorbol esters. The contribution of each individual PKC isozyme to malignant transformation is only partially understood, but it is clear that each PKC plays a different role in cancer progression. PKC deregulation is a common phenomenon observed in breast cancer and PKC expression and localization are usually dynamically regulated during mammary gland differentiation and involution. Overexpression of several PKCs has been reported in malignant breast tissue and breast cancer cell lines (24). Since PKC deregulation is observed in breast cancer (25), this kinase family is a promising target for blocking or reverting breast cancer malignancy. PKC is known to have at least 10 phospholipid-dependent serine-threonine kinase isoforms, one of which is PKC 8 . The role of PKC $\delta$ in breast cancer remains ambiguous and limited information is available with regard to expression levels of PKC $\delta$ in primary tumors. Although altered PKC $\delta$ expression does not appear to be a prerequisite for breast cancer progression, specific previous studies, including our own, have identified a protumorigenic role for PKCס overexpression in murine mammary cells via the induction of survival and anchorage-independent growth (26). PKC $\delta$ has been reported to promote proliferation (27) and metastasis development (28), whereas its depletion is sufficient to drive murine mammary cancer cells into apoptosis (29). By contrast, several studies have revealed that PKC $\delta$ mediates antiproliferative responses. For example, the antimitogenic effect of inositol hexaphosphate in MCF-7 human breast cancer cells, which involves inhibition of ERK and Akt as well as pRb hypophosphorylation, is mediated by PKC $\delta$ (30). Crosstalk between PKC $\delta$ and ER has also been hypothesized. Of note, ER-positive breast cancer cell lines express high levels of PKC $\delta$ and are associated with an improved endocrine response, whereas ER-negative breast cancer cell lines express low PKC levels (31). Moreover, PKCd is likely to play a crucial role in antiestrogen resistance in breast cancer cells and has been associated with acquired resistance to TAM in patients with breast cancer (32). Based on these studies, we hypothesize that the PKC pathway is one of the most important pathways associated with inhibition of MCF-7 cell proliferation by TAM. To investigate this hypothesis, the signal transduction reporter vector was analyzed in MCF-7 cells stimulated by TAM.

pAP-1(PMA)-TA-luc is a signal transduction reporter vector. The vector contains multiple copies of the AP-1 enhancer, located upstream of luciferase, that responds specifically to phorbol ester treatment. Activating the PKC pathway by adding phorbol esters, including PMA, results in transcription factors binding AP-1 elements on the vector. It is designed for monitoring the induction of the PKC signal transduction pathway. AP-1 is a heterodimeric transcription factor that is composed of various members of the Jun and Fos families and binds to DNA at specific AP-1 binding sites. AP-1 transcriptional activity is increased by phosphorylation at serine residues in the c-Jun component of AP-1. In this study, no significant difference in c-Jun transcript and protein levels was identified in MCF-7 and MDA-MB-435 cells stimulated by TAM for $48 \mathrm{~h}$. In addition, transcriptional reporter gene assays indicated that TAM is an AP-1(PMA)-associated activator (Fig. 3A). These data indicate that TAM may function in a similar manner to PMA to modulate c-Jun activity through the PKC pathway in MCF-7 cells. To determine the role of c-Jun in antiproliferation of MCF-7 cells stimulated by TAM, the correlation between inhibition rates of MCF-7 cells and c-Jun expression and stimulation of TAM was analyzed. Inhibition rates of TAM-stimulated MCF-7 cells were revealed to be positively regulated by c-Jun overexpression and negatively regulated by c-Jun underexpression.

Overall, these results indicate that TAM inhibits proliferation of MCF-7 and MDA-MB-435 cells and has no statistically significant effect on c-Jun transcript and protein levels. However, TAM-stimulated antiproliferation of MCF-7 cells is positively regulated by c-Jun through activation of the PKC pathway.

\section{References}

1. Clarke M: Meta-analyses of adjuvant therapies for women with early breast cancer: the Early Breast Cancer Trialists' Collaborative Group overview. Ann Oncol 17 (Suppl 10): x59-x62, 2006.

2. Fisher B, Costantino JP, Wickerham DL, Redmond CK, Kavanah M, Cronin WM, Vogel V, Robidoux A, Dimitrov N, Atkins J, Daly M, Wieand S, Tan-Chiu E, Ford L and Wolmark N: Tamoxifen for prevention of breast cancer: report of the National Surgical Adjuvant Breast and Bowel Project P-1 Study. J Natl Cancer Inst 90: 1371-1388, 1998.

3. Katzenellenbogen BS, Fang H, Ince BA, Pakdel F, Reese JC, Wooge $\mathrm{CH}$ and Wrenn CK: William L. McGuire Memorial Symposium. Estrogen receptors: ligand discrimination and antiestrogen action. Breast Cancer Res Treat 27: 17-26, 1993.

4. Katzenellenbogen BS, Montano MM, Le Goff P, Schodin DJ, Kraus WL, Bhardwaj B and Fujimoto N: Antiestrogens: mechanisms and actions in target cells. J Steroid Biochem Mol Biol 53: 387-393, 1995.

5. Charlier C, Chariot A, Antoine N, Merville MP, Gielen J and Castronovo V: Tamoxifen and its active metabolite inhibit growth of estrogen receptor-negative MDA-MB-435 cells. Biochem Pharmacol 49: 351-358, 1995.

6. Amin S, Kumar A, Nilchi L, Wright K and Kozlowski M: Breast cancer cells proliferation is regulated by tyrosine phosphatase SHP1 through c-jun N-terminal kinase and cooperative induction of RFX-1 and AP-4 transcription factors. Mol Cancer Res 9: 1112-1125, 2011.

7. Mamay CL, Mingo-Sion AM, Wolf DM, Molina MD and Van Den Berg CL: An inhibitory function for JNK in the regulation of IGF-I signaling in breast cancer. Oncogene 22: 602-614, 2003. 
8. Montano MM and Katzenellenbogen BS: The quinone reductase gene: a unique estrogen receptor-regulated gene that is activated by antiestrogens. Proc Natl Acad Sci USA 94 2581-2586, 1997.

9. Umekita Y, Ohi Y, Sagara Y and Yoshida H: Overexpression of cyclinD1 predicts for poor prognosis in estrogen receptor-negative breast cancer patients. Int J Cancer 98: 415-418, 2002.

10. Altucci L, Addeo R, Cicatiello L, Dauvois S, Parker MG, Truss M, Beato M, Sica V, Bresciani F and Weisz A: 17beta-Estradio induces cyclin D1 gene transcription, p36D1-p34cdk4 complex activation and $\mathrm{p} 105 \mathrm{Rb}$ phosphorylation during mitogenic stimulation of G(1)-arrested human breast cancer cells. Oncogene 12: 2315-2324, 1996.

11. Fu XD, Cui YH, Lin GP and Wang TH: Non-genomic effects of 17beta-estradiol in activation of the ERK1/ERK2 pathway induces cell proliferation through upregulation of cyclin D expression in bovine artery endothelial cells. Gynecol Endocrino 23: 131-137, 2007.

12. Urtreger AJ, Kazanietz MG and Bal de Kier Joffe ED Contribution of individual PKC isoforms to breast cancer progression. IUBMB Life 64: 18-26, 2012.

13. Orchel A, Dzierzewicz Z, Parfiniewicz B, Weglarz L and Wilczok T: Butyrate-induced differentiation of colon cancer cells is PKC and JNK dependent. Dig Dis Sci 50: 490-498, 2005.

14. Liu DS, Krebs CE and Liu SJ: Proliferation of human breast cancer cells and anti-cancer action of doxorubicin and vinblastine are independent of PKC-alpha. J Cell Biochem 101: 517-528, 2007.

15. Li Z, Wang N, Fang J, Huang J, Tian F, Li C and Xie F: Role of PKC-ERK signaling in tamoxifen-induced apoptosis and tamoxifen resistance in human breast cancer cells. Oncol Rep 27: 1879-1886, 2012.

16. Stabel S and Parker PJ: Protein kinase C. Pharmacol Ther 51: 71-95, 1991

17. Newton AC: Protein kinase C: structure, function and regulation. J Biol Chem 270: 28495-28498, 1995.

18. Newton AC: Regulation of protein kinase C. Curr Opin Cell Biol 9: 161-167, 1997.

19. Fisher B, Dignam J, Bryant J, DeCillis A, Wickerham DL, Wolmark N, Costantino J, Redmond C, Fisher ER, Bowman DM, Deschênes L, Dimitrov NV, Margolese RG, Robidoux A, Shibata H, et al: Five versus more than five years of tamoxifen therapy for breast cancer patients with negative lymph nodes and estrogen receptor-positive tumors. J Natl Cancer Inst 88: 1529-1542, 1996.

20. Berstein LM, Zheng H, Yue W, Wang JP, Lykkesfeldt AE, Naftolin F, Harada H, Shanabrough M and Santen RJ: New approaches to the understanding of tamoxifen action and resistance. Endocr Relat Cancer 10: 267-277, 2003.

21. Santen RJ: Long-term tamoxifen therapy: can an antagonist become an agonist? J Clin Endocrinol Metab 81: 2027-2029, 1996.
22. Shanmugam M, Krett NL, Maizels ET, Cutler RE Jr, Peters CA, Smith LM, O'Brien ML, Park-Sarge OK, Rosen ST and Hunzicker-Dunn M: Regulation of protein kinase $C$ delta by estrogen in the MCF-7 human breast cancer cell line. Mol Cell Endocrinol 148: 109-118, 1999.

23. Keshamouni VG, Mattingly RR and Reddy KB: Mechanism of 17-beta-estradiol-induced Erk1/2 activation in breast cancer cells. A role for HER2 AND PKC-delta. J Biol Chem 277: 22558-22565, 2002.

24. Tanaka Y, Gavrielides MV, Mitsuuchi Y,Fujii T and Kazanietz MG: Protein kinase $\mathrm{C}$ promotes apoptosis in $\mathrm{LNCaP}$ prostate cancer cells through activation of p38 MAPK and inhibition of the Akt survival pathway. J Biol Chem 278: 33753-33762, 2003.

25. Jarzabek K, Laudanski P, Dzieciol J, Dabrowska M and Wolczynski S: Protein kinase $\mathrm{C}$ involvement in proliferation and survival of breast cancer cells. Folia Histochem Cytobiol 40: 193-194, 2002.

26. Liu JF, Crepin M, Liu JM, Barritault D and Ledoux D: FGF-2 and TPA induce matrix metalloproteinase-9 secretion in MCF-7 cells through PKC activation of the Ras/ERK pathway. Biochem Biophys Res Commun 293: 1174-1182, 2002.

27. Grossoni VC, Falbo KB, Kazanietz MG, de Kier Joffe ED and Urtreger AJ: Protein kinase $\mathrm{C}$ delta enhances proliferation and survival of murine mammary cells. Mol Carcinog 46: 381-390, 2007.

28. Kiley SC, Clark KJ, Duddy SK, Welch DR and Jaken S: Increased protein kinase $\mathrm{C}$ delta in mammary tumor cells: relationship to transformation and metastatic progression. Oncogene 18: 6748-6757, 1999

29. Lonne GK, Masoumi KC, Lennartsson J and Larsson C: Protein kinase Cdelta supports survival of MDA-MB-231 breast cancer cells by suppressing the ERK1/2 pathway. J Biol Chem 284: 33456-33465, 2009.

30. Vucenik I, Ramakrishna G, Tantivejkul K, Anderson LM and Ramljak D: Inositol hexaphosphate (IP6) blocks proliferation of human breast cancer cells through a PKCdelta-dependent increase in $\mathrm{p} 27 \mathrm{Kip} 1$ and decrease in retinoblastoma protein (pRb) phosphorylation. Breast Cancer Res Treat 91: 35-45, 2005.

31. Assender JW, Gee JM, Lewis I, Ellis IO, Robertson JF and Nicholson RI: Protein kinase $\mathrm{C}$ isoform expression as a predictor of disease outcome on endocrine therapy in breast cancer. J Clin Pathol 60: 1216-1221, 2007.

32. Nabha SM, Glaros S, Hong M, Lykkesfeldt AE, Schiff R, Osborne K and Reddy KB: Upregulation of PKC-delta contributes to antiestrogen resistance in mammary tumor cells. Oncogene 24: 3166-3176, 2005. 